\title{
Spontaneous Uphill Movement and Self-Removal of Condensates on
}

\section{Hierarchical Tower-Like Arrays}

Min $\mathrm{He}^{1}$ *, Yue Ding ${ }^{1}$, Jing Chen ${ }^{2}$, Yanlin Song ${ }^{1}$ *

1 Key Laboratory of Green Printing, Institute of Chemistry, Chinese Academy of

Sciences, Beijing National Laboratory for Molecular Sciences, Beijing 100190, China

2 Department of Chemistry, School of Science, Tianjin University of Science and Technology, Tianjin, 300457 (P.R. China)

Corresponding E-mail: ylsong@iccas.ac.cn, heminyiwen@iccas.ac.cn.

Author contribution: Min He and Yue Ding contributed equally. 


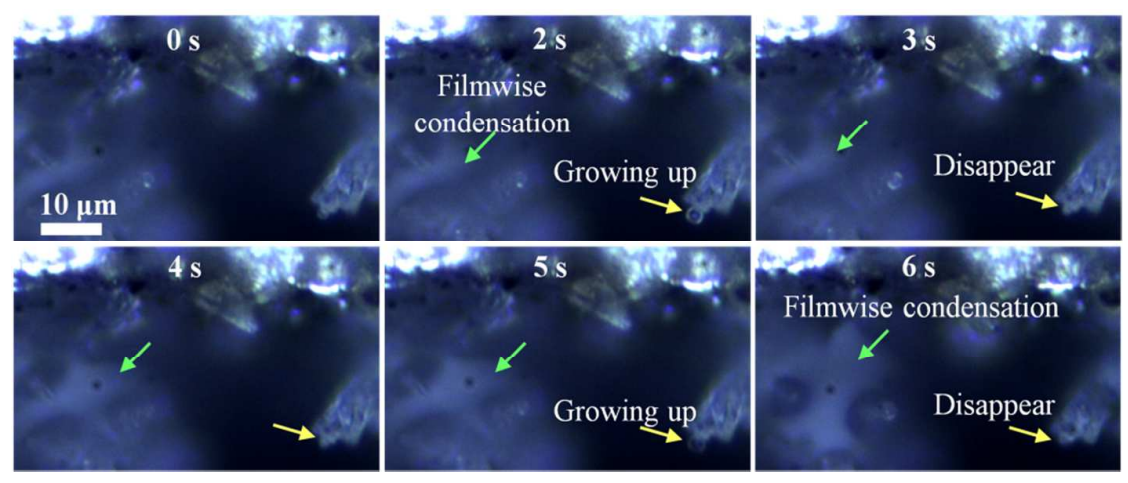

Figure S1. Condensation on bare arrays without being modified by PDMS, green arrows show the filmwise condensation, yellow arrows show the formation, growth and disappearance of tiny condensed droplets. 


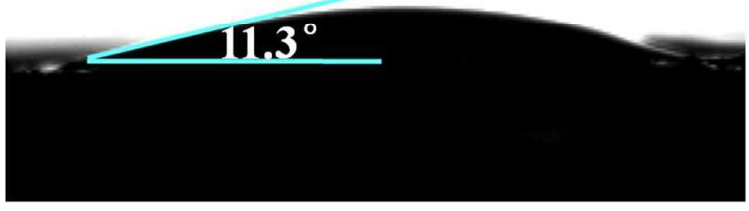

Figure S2. A water drop on the bare tower-like structure, the average contact angle of droplets on such surface is $11.3^{\circ}$. 
The Laplace pressure driving condensed droplets movinguphill to the top of tower-like structures

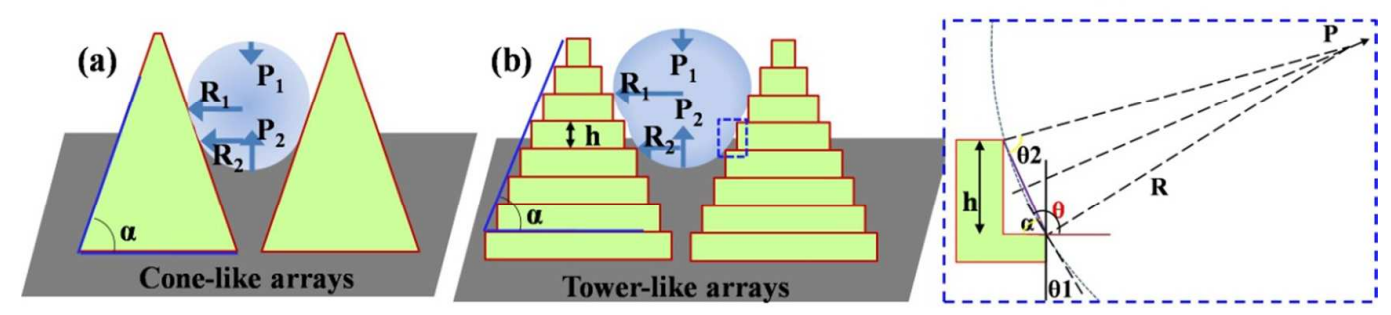

Figure S3. Scheme of a micro-droplet suspending on neighboring cone-like structures

(a) and hierarchical towers (b).

The Laplace pressure $(\mathrm{P})$ from the ratchet-like sidewall is

$$
\mathrm{P}=\frac{-2 \gamma}{\mathrm{R}}
$$

$\mathrm{R}$ is the radius of curvature, $\gamma$ is the water surface tension.

In Figure S1 (a), the total Laplace pressure for a droplet entrapped in two neighboring cone-like structures is

$$
\mathrm{P}^{\text {total }}=-2 \gamma\left(\frac{1}{\mathrm{R}_{2}}-\frac{1}{\mathrm{R}_{1}}\right) \cos \theta
$$

In Figure S1 (b), The upward component of $\mathrm{P}$ for a dropletentrapped in two neighboring hierarchical towersis

$$
\mathrm{P}_{\mathrm{up}}=\mathrm{P} \cos \alpha
$$

$\alpha$ is the tilted angle of a tower-like structure,

$$
\mathrm{L}=2 \mathrm{R} \cos \theta_{2}
$$

and

$$
\mathrm{L}=\frac{\mathrm{h}}{2 \sin \alpha}
$$


$\mathrm{L}$ is the length of the line connecting neighboring nano-plates' edge, $\mathrm{h}$ is the thickness of a single plate.

With simple geometric operation, we obtain:

$$
\begin{gathered}
\theta_{2}=\pi-\alpha-\theta_{1} \\
\theta_{1}=\theta-\frac{\pi}{2}
\end{gathered}
$$

$\theta_{1}$ is the angle between the tangent of curved water surface and the perpendicular direction, $\theta_{2}$ is the angle between the normal line and the line connecting neighboring nano-plates' edge, $\theta$ is the apparent CA of water droplets on the surface.

Combining equations (1)-(5) yields an expressionthat relates the Laplace pressure and the surface properties

$$
\mathrm{P}_{\mathrm{up}}=\frac{-4 \gamma \sin (2 \alpha) \cos \left(\alpha+\theta_{1}\right)}{\mathrm{h}}
$$

Thus, the total Laplace pressure driving condensates moving uphill to the top of microtowers is

$$
\mathrm{P}_{\text {up }}^{\text {total }}=2 \gamma\left(\frac{1}{\mathrm{R}_{1}}-\frac{1}{\mathrm{R}_{2}}\right) \cos \theta+\mathrm{n} \sum_{\mathrm{i}=1}^{\mathrm{j}} \mathrm{P}_{\mathrm{up}}
$$

with

$$
j=\frac{\left(R_{1}-R_{2}\right) \tan \alpha}{h}
$$

here $0 \leq \theta \leq \alpha$

$\mathrm{R}_{1}$ is the half pitch of nano-plates on neighboring tower-like structures contacting with the up curved water surface, $\mathrm{R}_{2}$ is the half pitch of nano-plates on neighboring tower-like structures contacting with the bottom curved water surface, $\mathrm{n}$ is the number 
of tower-like structures contacting with a microdroplet, $\mathrm{j}$ is the number of nano-plates on a single tower-like structure contacting with a microdroplet.

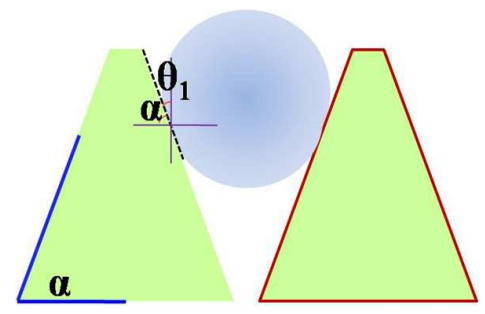

Figure S4. Condensed droplet suspends between two cone-like structures.

On the cone-like structures, the $\left(\alpha+\theta_{1}\right)$ is equal to $\pi / 2$ and consequently the cosine of $\left(\alpha+\theta_{1}\right)$ is equal to 0 (Figure S4). As a result, the equation (9) can be reduced to the equation (2) which is applied to cone-like structures as mentioned above.

By comparison the Laplace pressure on the two surfaces, we obtain

$$
\frac{(\text { tower }) \mathrm{P}_{\text {up }}^{\text {total }}}{(\text { cone }) \mathrm{P}_{\text {up }}^{\text {total }}}=1+\frac{\mathrm{n} \sum_{\mathrm{i}=1}^{\mathrm{j}} \mathrm{P}_{\mathrm{up}}}{2 \gamma\left(\frac{1}{\mathrm{R}_{1}}-\frac{1}{\mathrm{R}_{2}}\right) \cos \theta}
$$

Combining equation (6), (7) and (10), the following equation is obtained

$$
\frac{(\text { tower }) \mathrm{P}_{\text {up }}^{\text {total }}}{(\text { cone }) \mathrm{P}_{\text {up }}^{\text {total }}}=1+\frac{\mathrm{n} \sum_{\mathrm{i}=1}^{\mathrm{j}} \mathrm{R}_{1} \mathrm{R}_{2} \sin (2 \alpha) \cos (\alpha+\theta)}{\left(\mathrm{R}_{1}-\mathrm{R}_{2}\right) \mathrm{h} \cos \theta}
$$

The ratio of Laplace pressure on tower-like structures and cone-like structures suggests the advantage of tower-like structures in uphill movement of condensates. Thus, maximum values of the $\theta_{\mathrm{i}}$ can be determined by the first and second derivative of eq. 11 with respect to $R_{2}$. The result show when $R_{1}$ is 2 times of $R_{2}$ in the case of $h$ is $\sim 100 \mathrm{~nm}$, the maximum $\mathrm{R} 1$ is $\sim 2.4 \mu \mathrm{m}$ (the pitch of two neighboring microtowers), $\theta$ is $108^{\circ} . \alpha$ is calculated to be $\sim 72.6^{\circ}$, the Laplace pressure on the tower-like structures is $\sim 30$ times larger than that on the cone-like structures. 


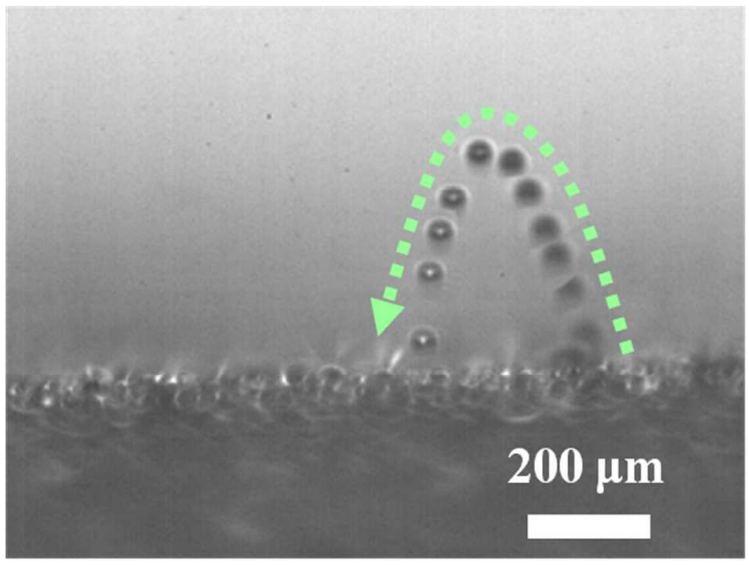

Figure S5. An overlapped optical side-view showing the trajectory of a merged micro-droplet jumping from the horizontal $\mathrm{S}_{\mathrm{t}}$. Dashed green arrow indicates the jump and fall of condensed droplets. 